\title{
Cadena Láctea Argentina: Un análisis estructural de derivación de demandas intermedias para la obtención de las elasticidades
}

\section{Argentine Dairy Chain: A structural analysis of the derivation of intermediate demands to elasticities acquisition}

Jimena Vicentin Masaro' (1)

'Instituto de Economía Aplicada Litoral, Facultad de Ciencias Económicas, Universidad Nacional del Litoral (UNL), Santa Fe (SF), Argentina. E-mail: jime.v.masaro@gmail.com

\begin{abstract}
Como citá: Vicentin Masaro, J. (2022). Cadena Láctea Argentina: un análisis estructural de derivación de demandas intermedias para la obtención de las elasticidades. Revista de Economia e Sociologia Rural, 60(1), e234604. https://doi. org/10.1590/1806-9479.2021.234604
\end{abstract}

\begin{abstract}
Resumen: Atendiendo a la importancia de la cadena productiva láctea en la economía argentina, se realiza análisis estructural de la misma derivando las demandas intermedias para conocer las elasticidades. A partir de un estricto planteamiento teórico de la cadena y las relaciones entre los diferentes niveles que lo componen y, utilizando un modelo de ecuaciones simultáneas, se cuantifican las relaciones de precios y cantidades a cada nivel para evaluar los efectos de posibles cambios en las variables claves de ésta cadena productiva, entre ellos de los precios. Se utiliza información secundaria, y se estima el modelo mediante mínimos cuadrados en tres etapas. Como resultados se destaca la inelasticidad de la oferta y demanda de todos los niveles respecto a sus propios precios, y mayor sensibilidad al precio mayorista. De las variables de política, solo los impuestos a las exportaciones resultan significativas. Cabe mencionar que si bien la no incorporación de stocks y la utilización de datos agregados es una limitación del presente, el aporte fundamental radica en el diseño integral de la cadena argentina con el tratamiento simultáneo de la determinación de las cantidades y precios, permitiendo conocer las relaciones a lo largo de dicha cadena de modo integral.
\end{abstract}

Palabras clave: cadena productiva. elasticidades. SEM. Argentina.

\begin{abstract}
Considering the dairy chain's relevance to the Argentine economy, a structural analysis was carried out deriving the intermediate demands to know the elasticities of this chain. A formal theoretical approach to the chain and its different levels of relationships is defined. Then, using a model of simultaneous equations, each level price and relationship quantities were quantified to evaluate the possible effects of keys variables changes along the whole chain, including prices. Secondary information is used, and the model was estimated by three-stage least squares. It was found that supply and demand are self-price inelastic at all levels, but greater sensitivity to the wholesale price. Among policy variables considered, only taxes on exports are significant. The lack of stock variables and the aggregate data usage is a limitation of this work, but the originality lies in the integral design of the Argentine chain with the simultaneous treatment of the determination of the quantities and prices, allowing to know the relationships along this chain in a complete way.
\end{abstract}

Keywords: productive chain, elasticities, SEM, Argentina.

\section{Introducción}

El sector lácteo ocupa un lugar relevante dentro de las actividades agroindustriales (European Association of Dairy Trade, 2020). Los mayores productores de leche son la Unión Europea, India y Estados Unidos y, cada cadena productiva posee una estructura de funcionamiento con características, forma de organización y relaciones entre agentes que son únicas, influyendo la idiosincrasia del mercado al que abastece así como la legislación impositiva y normativa de cada país. 
Muchos trabajos científicos a nivel internacional analizan la dinámica de sectores productivos agroindustriales algunas veces con objetivos académicos y otros más prácticos (e.g. Cakir \& Balagtas, 2012; Herrera Ramírez et al., 2018; Khosravi \& Esfandabadi, 2018; Sertoglu et al., 2017). De los aplicados a cadenas lácteas, los objetivos varían entre el análisis sobre la evolución de cantidades comercializadas (Lie et al., 2018; Salou et al., 2017; Yu, 2012) factores determinantes por nivel productivo (Nipers et al., 2017; Santeramo, 2014; Zhang \& Li, 2015); determinación de precios (Bolotova, 2017; Eičaite \& Mikelionytė, 2017) y, efectos de políticas (Kumar et al., 2019; Paterson \& Holden, 2019), por mencionar algunos. Una gran parte de estos trabajos utiliza los modelos de ecuaciones simultáneas estáticas y dinámicas (SEM y DSEM, respectivamente) ${ }^{1}$ como metodología de análisis, para incorporar el conocimiento estructural que tienen de la cadena productiva en particular.

Si bien la cadena láctea argentina no es la más importante a nivel mundial, si lo es a nivel regional. Es la tercera actividad de importancia dentro del sector agroindustrial del país; tiene una producción primaria concentrada en unas pocas provincias, ${ }^{2}$ ubicándose en el décimo primer puesto a nivel mundial en 2018. La industria, es la tercera en importancia dentro del sector alimenticio y posee gran heterogeneidad en la estructura tecnológica. Las exportaciones representan entre un $20-25 \%$ de la leche cruda procesada, y el resto se destina a mercado doméstico cuya comercialización está altamente concentrada en el canal supermercadista ${ }^{3}$ (Cardin \& Iturregui, 2016; Castro, 2017; Guerrero \& Lilia, 2016; Petrecolla, 2016).

Esta cadena productiva ha sido objeto de diferentes estudios para comprender su funcionamiento, algunos con enfoques científicos y otros técnicos. De los primeros, muchos han tratado de describir los comportamientos económicos de la cadena, pero lo hacen de manera pragmática, sin articular los niveles. En particular, ninguno ha logrado cuantificar el impacto de factores claves sobre precios y cantidades teniendo en cuenta la integralidad de la misma. Las razones probablemente sean la escasez de datos y dificultad para conseguirlos, pero sin embargo, este tipo de análisis tiene múltiples ventajas. Es una herramienta para conocer la relevancia de cada variable sobre la actividad de un modo integral, así como para predecir y el analizar escenarios realistas que mejoren la toma de decisiones tanto de agentes públicos como privados.

Por tanto, este trabajo tiene el objetivo de cuantificar las relaciones de precios y cantidades a cada nivel de la cadena para evaluar los efectos de los cambios en variables claves, reconociendo la integralidad de dicha cadena. El diseño de oferta y demanda específicamente aplicada a la estructura particular de la cadena teniendo en cuenta sus relaciones verticales y bajo un sistema de demandas derivadas es el principal aporte del trabajo. Además, la aplicación y sus consecuentes resultados que se visualizan como superadores en términos de los antecedentes encontrados.

A tal fin, el artículo comienza con la exposición de la problemática y luego, del marco teórico. Posteriormente, se muestra la estrategia metodológica así como los datos utilizados en la aplicación práctica. Posteriormente le siguen los resultados conjuntamente con la discusión y, luego, las conclusiones. Para aquellos lectores especializados se pone a disposición la derivación teórica y metodológica como complemento en los Anexos 1 y 2, respectivamente.

\section{Modelo Económico}

Se utiliza el concepto de "cadena productiva", es decir, niveles productivos interconectados cuyos precios se determinan en la interacción de la oferta y demanda. Estos precios son

\footnotetext{
1 Por sus siglas en inglés Structural Equation Model y Dynamic Structural Equation Model.

2 Santa Fe, Córdoba, Buenos Aires, Entre Ríos y La Pampa acumulan el 85\% de la producción.

3 Acumulan el $50 \%$ de estas ventas.
} 
señales transmitidas a lo largo de la cadena, que constituyen incentivos de la cantidad y mix de productos que son ofrecidos en cada nivel. La demanda al interior de la cadena es derivada de lo que ocurre en el resto, y los precios que se pagan a lo largo de la misma también están influenciados por la situación y el desempeño del conjunto de los eslabones.

Pero además de los precios, existen otras variables que influyen sobre la decisión de los agentes y su desempeño productivo así como en el agregado de cada nivel. Algunas de estas variables corresponden a la estructura de costos, e.g. los insumos y la tecnología (que a corto plazo suele ser exógena), y otras son externas a los agente decisorios como por ejemplo, las condiciones políticas, biológicas, o eventos naturales, entre otros (Ibarra Salazar \& García Pérez, 2016).

Para formalizar el modelo sobre la estructura de la cadena láctea argentina, se considera que la industria láctea (manufactura) puede vender en el exterior y al mercado interno (o minorista), pero no es formador de precios en el primero (país tomador de precios). En segundo lugar, no hay inventarios en ninguno de los niveles. Aunque este supuesto es plausible para el caso de la producción primaria, es restrictivo para el resto de la cadena. Sin embargo, se simplifica por falta de datos sobre stocks en la aplicación particular. En el Anexo 1 se expone un mayor detalle de la derivación del modelo teórico estructural que aquí se presenta.

Así, en el nivel primario la cantidad ofrecida de leche cruda proviene de la decisión óptima de los productores tamberos. Suponiendo un productor representativo con tecnología tipo Cobb-Douglas, donde $z_{\mathrm{il}}$ son los insumos en la producción de leche $\left(\mathrm{i}=1, \ldots, \mathrm{n}_{1}\right)$ cuyos precios son $\mathrm{w}_{11}, \ldots, \mathrm{w}_{\mathrm{n}_{1}}$ y $\mathrm{p}_{1} \mathrm{el}$ precio de la leche cruda (único producto obtenido en este nivel productivo). Entonces el productor resuelve el problema de maximización de los beneficios, obteniendo las demandas de los factores y, con éstas, la oferta de leche cruda. Linealizando la oferta de leche cruda (ecuación 8) se tiene:

$\ln \mathrm{q}_{1}^{\mathrm{s}}=\lambda_{0,1}^{\mathrm{s}}+\sum_{\mathrm{i}=1}^{\mathrm{n}_{1}} \lambda_{\mathrm{i}, 1} \ln \mathrm{w}_{\mathrm{i}}+\lambda_{\mathrm{p}, 1} \ln \mathrm{p}_{1}$

donde $\lambda_{\mathrm{p}, 1}$ es la elasticidad precio-propio de la oferta de leche cruda, $\mathrm{y} \lambda_{\mathrm{i}, 1}$ l las elasticidades de oferta respecto a los precios de los $n_{l}$-ésimos insumos de la producción primaria.

Un productor mayorista industrial, por su parte, produce dos tipos de bienes, a saber, el que vende en el mercado interno y el que coloca en el externo (simbolizados con los sub-índices $m$ $y$ *, respectivamente). Los factores de producción que dicho productor utiliza se puede dividir en cantidad de leche cruda $\left(\mathrm{q}_{1}^{\mathrm{d}}\right)$ y otros insumos $\left(\mathbf{z}_{\mathrm{M}}{ }^{4}\right.$ el subíndice $M$ indica nivel mayorista),

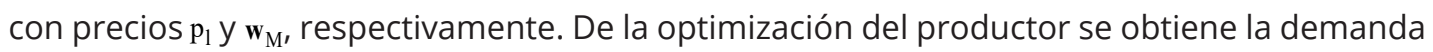
de factores -en la cual está la demanda derivada de leche cruda $\left(\mathrm{q}_{1}^{\mathrm{d}}\right)$ - $\mathrm{y}$, la oferta de productos lácteos $\left(\mathbf{q}_{\mathrm{m}}^{\mathrm{s}} \mathrm{Y} \mathbf{q}^{\mathrm{s}}\right)$. Esta última, puede ser tratada en litros equivalentes (Ite) sin perder generalidad, y por tanto, se simbolizan $\mathrm{q}_{\mathrm{m}}^{\mathrm{s}} \mathrm{y} \mathrm{q}_{*}^{\mathrm{s}}$. Las versiones linealizadas de estas funciones (ecuaciones 10 , 11 y 13 del Anexo 1) son,

$$
\begin{aligned}
& \ln \mathrm{q}_{\mathrm{m}}^{\mathrm{s}}=\tilde{\lambda}_{0, \mathrm{~m}}^{\mathrm{s}}+\gamma_{\mathrm{A}_{\mathrm{m}}} \ln \mathrm{A}_{\mathrm{m}}+\gamma_{\mathrm{m}, *} \ln \mathrm{p}_{*}+\gamma_{\mathrm{m}, \mathrm{m}} \ln \mathrm{p}_{\mathrm{M}}, \\
& \ln \mathrm{q}_{*}^{\mathrm{s}}=\tilde{\lambda}_{0, *}^{\mathrm{s}}+\gamma_{\mathrm{A}_{\mathrm{m}}} \ln \mathrm{A}_{\mathrm{m}}+\gamma_{*, *} \ln \mathrm{p}_{*}+\gamma_{*, \mathrm{~m}} \ln \mathrm{p}_{\mathrm{M}} . \\
& \ln \mathrm{q}_{\mathrm{l}}^{\mathrm{d}}=\lambda_{0,1}^{\mathrm{d}}+\prod_{\mathrm{k}=1}^{\mathrm{n}_{\mathrm{m}}} \delta_{\mathrm{k}, \mathrm{w}_{\mathrm{m}}} \ln \mathrm{w}_{\mathrm{km}}+\delta_{\mathrm{l}}\left(\delta_{\mathrm{l}}-1\right) \ln \mathrm{p}_{\mathrm{l}}+\tau_{\mathrm{m}} \ln \mathrm{p}_{\mathrm{M}}+\tau_{*} \ln \mathrm{p}_{*} .
\end{aligned}
$$

donde se muestran las elasticidades correspondientes a cada variable de interés.

\footnotetext{
4 Los valores en negrita simbolizan matrices.
} 
En el nivel de comercialización interna, los productos son distribuidos en el mercado interno por agentes que, demandan a su vez al nivel mayorista los productos como insumos. Estos productos no sufren una transformación física pero si un servicio de distribución. Dividiendo los costos en productos lácteos comprado a los industriales $\left(\mathrm{q}_{\mathrm{m}}\right)$ a un precio $\mathrm{p}_{\mathrm{M}}$, y otros insumos $\left(\mathrm{z}_{\mathrm{if}}\right.$, con $\left.\mathrm{i}=1 \ldots \mathrm{n}_{\mathrm{f}}\right)$ cuyos precios son $\mathrm{w}_{\mathrm{lf}}, \ldots, \mathrm{w}_{\mathrm{n}_{\mathrm{f}}}$; suponiendo una tecnología de producción Cobb-Douglas y con $\mathrm{p}_{\mathrm{m}}$ el precio al que vende al mercado interno los productos, mediante la maximización de los beneficios el distribuidor obtiene la demanda de productos lácteos como insumo y a su vez, la oferta final a los consumidores (ecuaciones 14 y 15 del Anexo 1):

$\ln \mathrm{q}_{\mathrm{m}}^{\mathrm{d}}=\lambda_{\mathrm{o}, \mathrm{m}}^{\mathrm{d}}+\theta_{\mathrm{M}}^{\mathrm{d}} \ln \mathrm{p}_{\mathrm{M}}+\theta_{\mathrm{m}}^{\mathrm{d}} \ln \mathrm{p}_{\mathrm{m}}+\sum_{\mathrm{j}} \theta_{\mathrm{jf}}^{\mathrm{d}} \ln \mathrm{w}_{\mathrm{jf}}$,
$\ln \mathrm{q}_{\mathrm{cf}}^{\mathrm{s}}=\lambda_{\mathrm{o}, \mathrm{ef}}^{\mathrm{s}}+\theta_{\mathrm{M}}^{\mathrm{s}} \ln \mathrm{p}_{\mathrm{M}}+\theta_{\mathrm{m}}^{\mathrm{s}} \ln \mathrm{p}_{\mathrm{m}}+\sum_{\mathrm{j}} \theta_{\mathrm{jf}}^{\mathrm{s}} \ln \mathrm{w}_{\mathrm{jf}}$.

Al final de la cadena se encuentra la demanda final de los consumidores ( $q_{\mathrm{cf}}^{\mathrm{d}}$, en litros equivalentes), y suponiendo un consumidor representativo, quien demanda lácteos en el mercado minorista maximizando su utilidad a un nivel de ingreso. Así, siguiendo a Barnett \& Serletis, (2008) la demanda tradicional log-log es,

$\ln q_{\mathrm{cf}}^{\mathrm{d}}=\eta_{0, \mathrm{cf}}+\eta_{\mathrm{I}} \ln I+\eta_{\mathrm{m}, \mathrm{ef}} \ln \mathrm{p}_{\mathrm{m}}+\sum_{\mathrm{j}} \eta_{\mathrm{j}, \mathrm{cf}} \ln \mathrm{p}_{\mathrm{j}}$

con / como ingreso, $\eta_{\mathrm{I}}$ y $\eta_{\mathrm{m}, \mathrm{cf}}$ son la elasticidades ingreso y precio-propio no compensada (Cournot), respectivamente. La demanda externa se asume que está dada, y queda representada por el precio internacional.

\section{Materiales y Métodos}

De las ecuaciones del epígrafe 2 se observa directamente el papel que cumplen los precios de los diferentes niveles, pero además se incluyen otras variables relevantes teniendo en cuenta la teoría económica con respecto a funciones de beneficios (o utilidad) y la disponibilidad de información en el país de aplicación. En el Anexo 2 se detallan las condiciones en las que se introducen variables de interés en cada ecuación de oferta y demanda.

En el sistema completo, los precios y cantidades son endógenas (excepto los $\mathrm{p}_{*}$ ), se determinan simultáneamente y se supone equilibrio en cada nivel, i.e. $\mathrm{q}_{1}^{\mathrm{d}}=\mathrm{q}_{1}^{\mathrm{s}}, \mathrm{q}_{\mathrm{m}}^{\mathrm{d}}=\mathrm{q}_{\mathrm{m}}^{\mathrm{s}} \mathrm{y} \mathrm{q}_{\mathrm{cf}}^{\mathrm{d}}=\mathrm{q}_{\mathrm{cf}}^{\mathrm{s}}$. Si las ecuaciones de 1 a 7 se resuelven para las variables endógenas e incorporan las variables exógenas expuestas en el Anexo 2, se tiene:

$$
\begin{gathered}
\ln \mathrm{q}_{\mathrm{lt}}^{\mathrm{s}}-\gamma_{\mathrm{p}, 1} \ln \mathrm{p}_{\mathrm{lt}}=\alpha_{0,1}+\gamma_{1,1} \ln \mathrm{p}_{\mathrm{t}}^{\mathrm{soja}}+\gamma_{2,1} \ln \mathrm{p}_{\mathrm{t}}^{\text {maiz }}+\alpha_{1,1} \operatorname{Comp}_{\mathrm{t}} \\
+\alpha_{2,1} \operatorname{Clima}+\sum_{\mathrm{k}=2}^{4} \vartheta_{\mathrm{k}, 1} \mathrm{E}_{\mathrm{k}}+\mathrm{e}_{\mathrm{lt}}^{\mathrm{s}}, \\
\ln \mathrm{q}_{\mathrm{lt}}^{\mathrm{d}}-\tilde{\delta}_{\mathrm{l}} \ln \mathrm{p}_{\mathrm{lt}}-\tau_{\mathrm{M}} \ln \mathrm{p}_{\mathrm{Mt}}=\alpha_{0,1}^{\mathrm{d}}+\tau_{*} \ln \mathrm{p}_{* \mathrm{t}}+\alpha_{1,1}^{\mathrm{d}} \mathrm{t}+\mathrm{e}_{\mathrm{lt}}^{\mathrm{d}}, \\
\ln \mathrm{q}_{\mathrm{mt}}^{\mathrm{s}}-\gamma_{\mathrm{m}, \mathrm{m}} \ln \mathrm{p}_{\mathrm{Mt}}-\alpha_{5, \mathrm{~m}} \ln \mathrm{q}_{\mathrm{lt}}=\alpha_{0, \mathrm{~m}}+\gamma_{\mathrm{m}, *} \ln \mathrm{p}_{* \mathrm{t}}+\alpha_{2, \mathrm{~m}} \ln \operatorname{IBIF}_{\mathrm{t}}+ \\
+\alpha_{3, \mathrm{~m}} \operatorname{IPIM}_{\mathrm{t}}^{\mathrm{NG}}+\alpha_{4, \mathrm{~m}} \ln \operatorname{salario}_{\mathrm{t}}^{\text {Ind }}+\mathrm{e}_{\mathrm{mt}}^{\mathrm{s}},
\end{gathered}
$$




$$
\begin{aligned}
& \ln \mathrm{q}_{*_{\mathrm{t}}}^{\mathrm{s}}-\gamma_{*, \mathrm{~m}} \ln \mathrm{p}_{\mathrm{Mt}}-\alpha_{2, *} \ln \mathrm{q}_{\mathrm{lt}}=\alpha_{0, *}+\gamma_{*, *} \ln \mathrm{p}_{* \mathrm{t}}+\sum_{\mathrm{k}=2}^{4} \vartheta_{\mathrm{k}, *} \mathrm{E}_{\mathrm{k}}++\alpha_{1, *} \operatorname{Ret}_{\mathrm{t}}+\mathrm{e}_{* \mathrm{t}}^{\mathrm{s}}, \\
& \ln \mathrm{q}_{\mathrm{mt}}^{\mathrm{d}}-\theta_{\mathrm{M}}^{\mathrm{d}} \ln \mathrm{p}_{\mathrm{Mt}}-\alpha_{\mathrm{m}}^{\mathrm{d}} \ln \mathrm{p}_{\mathrm{mt}}=\alpha_{0, \mathrm{~m}}+\alpha_{1, \mathrm{~m}}^{\mathrm{d}} \mathrm{EMAE}_{\mathrm{t}}+\mathrm{e}_{\mathrm{mt}}^{\mathrm{d}}, \\
& \ln \mathrm{q}_{\mathrm{cf}_{\mathrm{t}}}^{\mathrm{s}}-\theta_{\mathrm{m}}^{\mathrm{s}} \ln \mathrm{p}_{\mathrm{mt}}-\theta_{\mathrm{M}}^{\mathrm{s}} \ln \mathrm{p}_{\mathrm{Mt}}=\alpha_{0, \mathrm{cf}}^{\mathrm{s}}+\alpha_{1, \mathrm{cf}}^{\mathrm{s}} \operatorname{IPC}_{\mathrm{t}}^{\mathrm{NG}}+\mathrm{e}_{\mathrm{cf}_{\mathrm{t}}}^{\mathrm{s}}, \\
& \ln \mathrm{q}_{\mathrm{cf}_{\mathrm{t}}}^{\mathrm{d}}-\eta_{\mathrm{m}, \mathrm{cf}} \ln \mathrm{p}_{\mathrm{mt}}=\alpha_{0, \mathrm{cf}}+\eta_{\mathrm{I}} \ln \text { salario }_{\mathrm{t}}+\alpha_{1, \mathrm{cf}} \mathrm{E}_{\mathrm{cf}_{\mathrm{t}}}+\mathrm{e}_{\mathrm{cf}_{\mathrm{t}}}^{\mathrm{d}} .
\end{aligned}
$$

Se utiliza la metodología de estimación Mínimo Cuadrados en Tres Etapas siguiendo a Zellner \& Theil (1962). Ésta contempla la correlación entre las co-variables con los errores y, además, la posibilidad de que éstos estén correlacionados contemporáneamente entre las ecuaciones. Luego, se realiza la validación del modelo por medio del análisis de los residuos: autocorrelación serial, heterocedasticidad y especificación (Pruebas Durbin-Watson, Breush-Pagan y Ramsey, respectivamente). Luego, se realiza la prueba Hausman para comprobar la existencia de correlaciones contemporáneas (Hausman, 1978). Y por último, se analiza la estacionariedad de los residuos de cada ecuación, los test Augmented Dickey Fuller(ADF).

\subsection{Variables y Fuentes}

En la Tabla 1 se muestran las variables con sus respectivas fuentes. Se cuenta con una base de datos mensual que va desde enero del 2000 hasta octubre del 2015, i.e. una longitud de 190 observaciones. Cabe mencionar que no es posible extender el período de análisis debido a la falta de estadísticas oficiales.

Algunas variables se utilizan en una versión modificada de la publicada en la fuente original, para más detalle ver Anexo 3.

Las variables en valores monetarios (\$ARG) son puestas en valores constantes de 2004, utilizando el IPC para deflactar los precios minoristas y sueldos; el IPIM para los valores en el nivel mayorista y de exportación y, en el nivel primario, se deflactan por medio del Índice de Precios al Productor (IPP).

De un análisis descriptivo de las variables puede constatarse que el promedio de las cantidades destinadas al mercado interno son superiores a las exportadas, y estas últimas, más variables. Con respecto a los precios, los minoristas son en promedio 30\% superior a los mayoristas.

\section{Resultados}

El modelo de ecuaciones simultáneas muestra un bien ajuste, con un $\mathrm{R}^{2}$ de McElroy fue significativo de 0.8493 . Por nivel se puede observar que las ecuaciones de oferta tienen un mejor ajuste que las demanda, exceptuando a la del nivel minorista (Tabla 2). Respecto de la validación general se puede constatar una correlación alta y significativa de los residuos entre ecuaciones, rechazando la independencia de las ecuaciones a lo largo de la cadena. Los residuos del modelo final no presentan problemas ni de auto-correlación serial ni de heterocedasticidad y son estacionarios.

En las columnas (a) y (d) de la Tabla 2 se muestran los resultados de la demanda y oferta del nivel primario, respectivamente. No se constata una dependencia significativa de demanda al precio-propio, lo cual puede deberse al hecho de que la industria procesa leche cruda y el pago lo hace con uno o dos meses de retraso, i.e. procesa antes de estar definido el precio final a abonar por la materia prima. 
Tabla 1. Variables y fuentes de la información.

\begin{tabular}{|c|c|c|c|c|}
\hline & Variables & $\begin{array}{l}\text { U. de } \\
\text { Medida }\end{array}$ & Modificada ${ }^{(3)}$ & Fuente \\
\hline \multirow{9}{*}{ 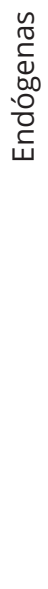 } & Cantidad de leche cruda & mill. Its. & No & Ministerio de Agroindustria (MiniAgri) \\
\hline & $\begin{array}{l}\text { Cantidad total mayorista (may) } \\
\text { de prod. lácteos }{ }^{(1)}\end{array}$ & mill. Ite. & $\mathrm{Si}$ & MiniAgri \\
\hline & $\begin{array}{l}\text { Cantidad minorista (min) final } \\
\text { del mix de prod. Lácteos }{ }^{(2)}\end{array}$ & mill. Ite. & $\mathrm{Si}$ & MiniAgri \\
\hline & $\begin{array}{l}\text { Cantidad may. mix de prod. } \\
\text { lácteos para el merc. interno }\end{array}$ & mill. Ite & $\mathrm{Si}$ & MiniAgri \\
\hline & $\begin{array}{l}\text { Cantidad exportada mix de prod. } \\
\text { lácteos }\end{array}$ & mill. Ite & $\mathrm{Si}$ & $\begin{array}{l}\text { Aduana, Penta Transaction, } \\
\text { MiniAgri }\end{array}$ \\
\hline & Precio de la leche cruda & $\begin{array}{l}\text { \$ constante } \\
\text { (cte)/lt }\end{array}$ & No & $\begin{array}{l}\text { MiniAgri, Instituto Provincial de } \\
\text { Estadísticas y Censos de Santa Fe (IPEC) }\end{array}$ \\
\hline & Precio may. mix de prod. lácteos & $\$$ cte/lte & $\mathrm{Si}$ & MiniAgri \\
\hline & Precio min. mix de prod. lácteos & $\$$ cte/lte & Si & MiniAgri \\
\hline & $\begin{array}{l}\text { Precio de exportación mix de } \\
\text { prod. lácteos }\end{array}$ & $\$$ cte/lte & $\mathrm{Si}$ & $\begin{array}{l}\text { Aduana, Penta Transaction, } \\
\text { MiniAgri, Banco Central de la } \\
\text { República Argentina (BCRA) }\end{array}$ \\
\hline \multirow{14}{*}{ 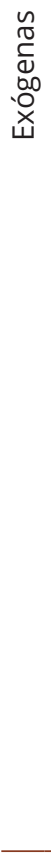 } & Precio del maíz & $\$$ cte/tn. & No & $\begin{array}{l}\text { Bolsa de Comercio de Rosario } \\
\text { (BCR) }\end{array}$ \\
\hline & Precio Interno de la soja & $\$$ cte/tn. & No & $\mathrm{BCR}$ \\
\hline & $\begin{array}{l}\text { Índice de Precios Interno al por } \\
\text { Mayor Nivel General (NG) }\end{array}$ & Base 2004. & No & $\begin{array}{l}\text { MiniAgri, Dirección Provincial de } \\
\text { Estadísticas y Censos de San Luis (DPEC) }\end{array}$ \\
\hline & Índice de Precios al Productor NG & Base 2004. & No & MiniAgri, DPEC \\
\hline & Índice de Precios al Consumidor NG & Base 2004. & No & $\begin{array}{l}\text { Instituto Nacional de Estadísticas y } \\
\text { Censos (INDEC), DPEC }\end{array}$ \\
\hline & $\begin{array}{l}\text { Estimador Mensual de Activ. } \\
\text { Económica (EMAE) }\end{array}$ & Base 2004. & No & INDEC \\
\hline & Índice de Salarios General & Base 2004. & No & INDEC \\
\hline & $\begin{array}{l}\text { Remuneración a Industria } \\
\text { Alimenticia }\end{array}$ & $\begin{array}{l}\text { \$ cte año } \\
2004 .\end{array}$ & $\mathrm{Si}$ & Ministerio de Economía, INDEC \\
\hline & Inversión Bruta Interna Fija (IBIF) & $\begin{array}{l}\text { \$ cte año } \\
2004 .\end{array}$ & No & INDEC \\
\hline & Retenciones a las exportaciones & Binarias & No & Depetris Guiguet et al. $(2011,2013)$ \\
\hline & $\begin{array}{l}\text { Compensaciones a prod. } \\
\text { primarios }\end{array}$ & Binarias & No & Depetris Guiguet et al. $(2011,2013)$ \\
\hline & Inundaciones y sequías & Binarias & No & Depetris Guiguet et al. $(2011,2013)$ \\
\hline & $\begin{array}{l}\text { Estacionalidad en producción } \\
\text { primaria/exportación }\end{array}$ & Binarias & $\mathrm{Si}$ & MiniAgri, Aduana. \\
\hline & $\begin{array}{l}\text { Estacionalidad en el consumo } \\
\text { min. }\end{array}$ & Binarias & Si & MiniAgri, Aduana. \\
\hline
\end{tabular}

Notas: (1) Es la suma de cantidades vendidas en el mercado minorista y externo. ${ }^{(2)}$ Las cantidades minoristas y mayoristas son idénticas, pero a efectos analíticos se mencionan por separado. ${ }^{(3)}$ Si la variable se modifica, se amplía en el Anexo 3. Fuente: elaboración propia

Respecto al comportamiento de la demanda ante cambios en los precios de productos elaborados, se observa que es inelástica a ambos precios, pero más a los mayoristas que a los de exportación. Un incremento del $10 \%$ de los primeros está acompañado por un aumento de $5 \%$ en la demanda de leche cruda, en cambio, el efecto es la mitad (2.5\%) si aumentan en los precios de exportación. Esto muestra la mayor importancia del mercado interno en toda la cadena, porque además, los precios mayoristas son, generalmente, superiores a los recibidos por exportación por lo que sus aumentos son más atractivos para los industriales (Depetris Guiguet et al., 2011).

Los coeficientes estimados en la oferta de leche cruda son los esperados y, exceptuando a las compensaciones, son significativos al nivel del $10 \%$. Se observa inelasticidad de la misma al propio precio lo que se puede deber a la imposibilidad las restricciones de la producción primaria a corto plazo. Se destaca la importancia de los precios de la soja en la oferta primera de leche, un aumento 
del $10 \%$ en el precio de la soja está acompañado por una disminución del 3.2\% de la oferta de leche aproximadamente, o que existiría sustitución de explotación en la actividad lechera.

Se observa, además, una alta dependencia del resto de las variables estacionales y de shocks climáticos, donde el mayor efecto proviene del primero, siendo el período nov-ene los meses que en promedio se ofrece mayor cantidad de leche cruda. Cabe destacar también la no significatividad de las políticas de compensaciones como medida para incentivar a la oferta, ya que dicho indicador no resulta significativo.

Los resultados de la demanda del nivel mayorista puede observarse en la columna (b) y, la de oferta en las (e) y ( $f$ ) de la Tabla 2, incluyendo en estas últimas a la oferta al mercado externo e interno mayorista, respectivamente. Se observa que la oferta mayorista es inelástica respecto al precio propio, en cambio la de exportaciones lo es tanto a los precios del mix de exportación como al mayorista, pero menos en éste último. Si las retenciones son un $5 \%$ del valor exportado, las exportaciones caen aproximadamente un $2.4 \%$, sin efectos sobre la oferta mayorista al mercado interno, dado que no es significativo el efecto de los precios de exportación en dicha ecuación. $\mathrm{Si}$, por otro lado, los precios mayoristas se incrementan 5\%, la cantidad de exportaciones caerían en $2.7 \%$ aproximadamente, mientras que las ofrecidas en el mercado interno aumentarían en 1.6\%. En el trabajo de García Arancibia et al. (2013) se obtienen unas diferencias de elasticidades menores al del presente trabajo, pero sólo en dicho momento sólo se modela el nivel industrial.

Ambas ofertas están influenciadas por las cantidades producidas de leche cruda en el nivel minorista, un incremento del $10 \%$ de dicha cantidad está asociado a un aumento de las exportaciones del 19\% y al mercado doméstico del 7.6\%, aproximadamente. Este mayor impacto relativo sobre las exportaciones tiene sentido ya que la proporción que se exporta (15-25\% promedio en lte) es significativamente menor a lo colocada en el mercado interno (Depetris Guiguet et al., 2011). Estos resultados son similares a los de García Arancibia et al. (2013) no sólo en términos relativos, sino también en los coeficientes.

Se destaca la no significancia de los salarios en la oferta mayorista, siendo esta industria una con los más altos costos laborales en el país (Observatorio de la Cadena Láctea Argentina, 2016). Estos resultados podrían deberse a que la variable usada no logra capturar el efecto (se usaron salarios de la industria alimenticia y no de la industria láctea) o que efectivamente las decisiones de producción se tomen con independencia del salario. Esta última hipótesis radica en la industria es altamente necesitada de la mano de obra para funcionar, los días de paro puedan ser mucho más costosos que pagar salarios más altos. Es necesario ahondar más en este punto en futuros estudios.

En las columnas (c) y (g) de la Tabla 2 se muestran las estimaciones de demanda y oferta minorista final del mix de lácteos, respectivamente. Con un ajuste individual en ambas ecuaciones no muy alto, se observa una demanda final muy inelástica al precio y al salario, resultado esperado ya que son productos de la canasta básica argentina. En un trabajo realizado por Fernández (2007), se encuentra una elasticidad precio similar a la encontrada en el presente trabajo pero la ingreso, inferior. Sin embargo, aunque dicho autor utiliza datos de hogares más precisos sobre preferencias sólo estudia demanda, sin tener en cuenta la cadena en conjunto. Cabe destacar las limitaciones de analizar demanda minorista a partir de valores agregados, sobre todo si se quiere estudiar preferencias.

Se observa que la oferta minorista es más inelástica al propio precio que al mayorista, esta última inversamente relacionada, i.e., un aumento del $10 \%$ del precio mayorista está asociado a una reducción en la oferta minorista del $2.5 \%$ y, una caída del $2.4 \%$ en la demanda de lácteos a nivel mayorista, por ser esta última una demanda derivada de la primera.

Otra variable de interés es el IPC, la cual involucra dos efectos en la oferta minorista: por un lado, referencia a otros costos distintos de los productos lácteos y, por el otro, la variación del precio del resto de los bienes que vende el oferente. En ambos casos los resultados son los esperados. En el primero porque un aumento de costos desincentiva a la oferta; y en el segundo, porque el aumento de precios de otros productos, dado un nivel del mix lácteo, los hace más atractivos para el oferente. Así, un aumento del $10 \% 5$ está asociado a una reducción de la oferta de $0.25 \%$ (aprox.), ceteris paribus.

${ }^{5}$ Son casi 25 puntos del IPC cuyo valor promedio del período es 243.3. 


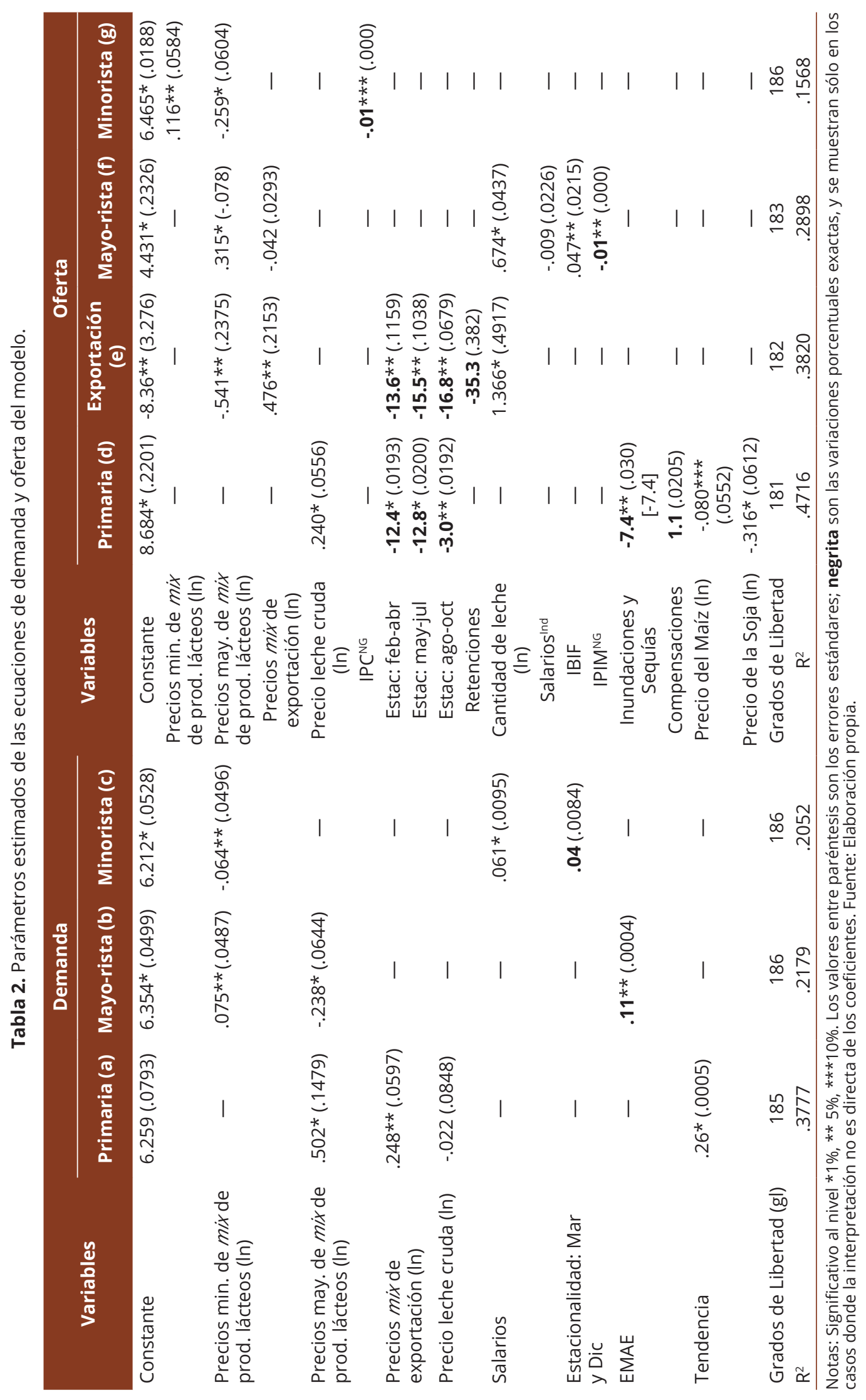




\section{Conclusiones}

Mediante la formalización de las relaciones a lo largo de la cadena láctea argentina con el objetivo de conocer la estructura integral misma, se cuantifican las relaciones de precios y cantidades a cada nivel para evaluar efectos de cambios en variables claves a lo largo de la misma, sobre todo de los precios. Cabe mencionar que, ante el estado del arte, el presente trabajo resulta un aporte original en modelar la cadena láctea de manera integral, i.e. teniendo en cuenta todos los niveles productivos. Se utiliza una metodología de regresión en tres etapas, y por medio de la compilación de una importante base de datos se loga estimar un sistema de ecuaciones simultáneas estructural.

Como resultados se puede destacar que, en el corto plazo, la oferta y demanda de todos los niveles es inelástica respecto a sus propios precios, con mayor sensibilidad al precio mayorista. La única variable política relevante es el impuesto a las exportaciones, que reduce el precio doméstico recibido y las cantidades exportadas. Los resultados presentados puestos en comparación con algunos trabajos aplicados sólo a ciertos niveles de la cadena argentina desarticulados, concuerdan sobre los efectos de las variables tenidas en cuenta con algunas diferencias absolutas.

En futuros trabajos sería necesario incorporar una dimensión dinámica a la estructura, ya que incorporaría más realismo al sistema, y mejor adaptación las condiciones particulares de esta actividad económica. Otro punto a considerar es la incorporación de stocks ya que si bien, la nulidad de esta variable se ajusta bien para la realidad del nivel primario, no así los subsiguientes niveles. Cabe mencionar que dicho supuesto se incorpora por motivos de escasez e inconsistencia de información oficial disponible. Habría que analizar la posibilidad de estimación de stocks.

El presente trabajo significa un importante esfuerzo en la obtención de información para la estructuración de este sistema, ya que en países como Argentina donde existe información deficiente, escasa, o discontinua es complicada la realización de estudios estructurales.

\section{Referencias}

Barnett, W. A., \& Serletis, A. (2008). Consumer preferences and demand systems. Journal of Econometrics, 147(2), 210-224.

Bolotova, Y. V. (2017). An analysis of milk pricing in the united states dairy industry. Agribusiness, 33(2), 194-208.

Cakir, M., \& Balagtas, J. V. (2012). Estimation Market Power of U.S. Dairy Cooperatives in the Fluid Milk Market. American Journal of Agricultural Economics, 94(3), 647-658.

Cardin, R., \& Iturregui, M. E. (2016). Informes de Cadenas de Valor. Láctea (Documento de Trabajo). Buenos Aires: Ministerio de Economía. Retrieved in 2020, Março 01, from https:// www.economia.gob.ar/peconomica/docs/Complejo_Lacteo.pdf

Castro, N. (2017). La dictadura de los supermercados: cómo los grandes distribuidores deciden lo que consumimos. Madrid: Ediciones Akal.

Depetris Guiguet, E., Rossini, G., García Arancibia, R., \& Vicentin Masaro, J. (2011). Competitividad del complejo lácteo santafesino. Santa Fe: Instituto de Economía Aplicada Litoral (IECAL), Universidad Nacional del Litoral.

Depetris Guiguet, E., Rossini, G., García Arancibia, R., Vicentin Masaro, J., \& Coronel, M. (2013). Cambio y tendencias en las políticas lecheras de los principales participantes del mercado 
internacional (2000-2012). Santa Fe: Instituto de Economía Aplicada Litoral (IECAL), Universidad Nacional del Litoral.

Eičaitè, O., \& Mikelionytè, D. (2017). Domestic dairy products: determination of market share on Internal Mrket of Lithuania. Scientific Papers. Series Management, Economic, Engineering in Agriculture and Rural Development, 17(3), 127-134.

European Association of Dairy Trade - Eucolait. (2020). Brussels, NL. Retrieved in 2020, Março 01, from http://www.eucolait.eu/

Fernández, S. J. (2007). Estimaciones de demanda de alimentos utilizando un sistema LINQUAD (Tesis de Grado). Universidad Nacional de Mar del Plata, Mar del Plata, Argentina. Retrieved in 2020, Março 01, from http://nulan.mdp.edu.ar/622/

García Arancibia, R., Depetris Guiguet, E., Vicentin Masaro, J., \& Rossini, G. E. (2013). Factores determinantes de la oferta exportadora láctea santafesina. SaberEs, 1(5), 67-82.

Greer, M. (2012). Electricity marginal cost pricing : Applications in eliciting demand responses. Waltham, MA: Elsevier.

Guerrero, C., \& Lilia, V. (2016). Análisis de Alternativas Comerciales para la Internacionalización de la producción láctea de la Asociación Productora y Comercializadora Agropecuaria Taya del Cantón Tulcán, provincia del Carchi (Trabajo de Titulación). Universidad Politécnica Estatal de Charchi. Tulcán, Ecuador.

Hausman, J. A. (1978). Specification tests in econometrics. Econometrica, 46(6), 1251.

Herrera Ramírez, M. H., Castro, J. O., Vargas, H. S. C., \& Martínez, M. A. (2018). Modelado dinámico y estratégico de la cadena agroindustrial de frutas : Un enfoque para la gestión de tecnología. Bogotá: Universidad Piloto de Colombia.

Ibarra Salazar, J., \& García Pérez, F. (2016). Las demandas de factores productivos en la industria maquiladora. Estudos Econômicos, 31(2), 265-303.

Khosravi, M., \& Esfandabadi, A. A. J. (2018). Simulating the effects of macroeconomic shocks on agricultural sector: Dynamic Stochastic General Equilibrium (DSGE) model approach. Majallah-i Tahqiqat-i Iqtisad va Tusiah-i Kishavarzi-i Iran, 48(4), 573-587.

Kumar, A., Mishra, A. K., Saroj, S., \& Joshi, P. K. (2019). Impact of traditional versus modern dairy value chains on food security: Evidence from India's dairy sector. Food Policy, 83, 260-270.

Lie, H., Rich, K. M., van der Hoek, R., \& Dizyee, K. (2018). An empirical evaluation of policy options for inclusive dairy value chain development in Nicaragua: A system dynamics approach. Agricultural Systems, 164, 193-222.

Nipers, A., Pilvere, I., \& Zeverte-Rivza, S. (2017). Projections for the latvian dairy and beef sector. Engineering for Rural Development, 24, 546-554.

Observatorio de la Cadena Láctea Argentina - OCLA. (2016). La competitividad de la Industria Láctea Argentina. Retrieved in 2020, Março 01, from http://www.ocla.org.ar/contents/ newswelcome/newsresearch/

Paterson, K. C., \& Holden, N. M. (2019). Assessment of policy conflict using systems thinking: A case study of carbon footprint reduction on Irish dairy farms. Environmental Science \& Policy, 101, 38-45.

Petrecolla, D. (2016). Estudio sobre las Condiciones de Competencia en el Sector Lechero de la República Argentina (Documento de Trabajo). Buenos Aires, Argentina: Ministerio de Agroindustria. Retrieved in 2020, Março 01, from http://www.ocla.org.ar/contents/news/ 
details/10013004-estudio-sobre-las-condiciones-de-competencia-en-el-sector-lechero-dela-republic

Salou, T., van der Werf, H. M. G., Levert, F., Forslund, A., Hercule, J., \& Le Mouël, C. (2017). Could EU dairy quota removal favour some dairy production systems over others? The case of French dairy production systems. Agricultural Systems, 153, 1-10.

Santeramo, F. G. (2014). On the estimation of supply and demand elasticities of agricultural commodites. Washington, DC: International Food Policy Research Institute.

Sertoglu, K., Sertoğlu, K., Ugural, S., \& Bekun, F. V. (2017). The contribution of agricultural sector on economic growth of Nigeria. International Journal of Economics and Financial Issues, $7(1), 547-552$.

Yu, X. (2012). Productivity, efficiency and structural problems in Chinese dairy farms. China Agricultural Economic Review, 4(2), 168-175.

Zellner, A., \& Theil, H. (1962). Three-Stage least squares: simultaneous estimation of simultaneous equations. Econometrica, 30(1), 54-78.

Zhang, Y., \& Li, C.-X. (2015). Analysis of the influence of the price factor on the yield of raw milk based on the VEC model. Heilongjiang Animal Science and Veterinary Medicine, 8(1), 1-4. 


\section{Anexo 1. Derivación del Modelo Económico}

En nivel primario y suponiendo un productor tambero representativo con tecnología tipo Cobb-Douglas, i.e. $q_{1}=A_{1} \prod_{i=1}^{n_{1}} z_{i l}^{\beta_{11}}$, siendo $z_{i l}$ los insumos en la producción de leche $\left(i=1, \ldots, n_{1}\right), \beta_{i l}$ los parámetros correspondientes $\left(\beta_{\mathrm{il}} \geq 0\right.$ y $\left.\sum_{\mathrm{i}=1}^{\mathrm{n}_{1}} \beta_{\mathrm{il}}<1\right)$ y, $\mathrm{A}_{1}$ es una constante tecnológica. Si $\mathrm{p}_{1}$ es el precio de la leche cruda (único producto del nivel productivo) y $\mathrm{w}_{11}, \ldots, \mathrm{w}_{\mathrm{n}_{1}} \mathrm{el}$ de los factores, el productor maximiza beneficios, obteniendo las demandas de los factores y con éstas, la oferta de leche cruda:

$$
\mathrm{q}_{1}^{\mathrm{s}}=\mathrm{B}_{1}\left(\mathrm{~A}_{1}, \beta_{11}, \ldots, \beta_{\mathrm{n}_{1}}\right) \mathrm{p}_{1} \exp \left(\frac{\sum_{\mathrm{j}} \beta_{\mathrm{jl}}}{1-\sum_{\mathrm{j}} \beta_{\mathrm{jl}}}\right) \prod_{\mathrm{i}=1}^{\mathrm{n}_{1}} \mathrm{w}_{\mathrm{i}} \exp \left(\frac{\beta_{\mathrm{il}}}{1-\sum_{\mathrm{j}} \beta_{\mathrm{jl}}}\right) .
$$

En el nivel mayorista, el productor industrial $(M)$ vende productos en el mercado interno $\mathrm{y} / \mathrm{o}$ en el externo ( $m \mathrm{y}^{*}$, respectivamente). Entonces se produce $\mathbf{q}_{\mathrm{M}}^{\mathrm{T}}=\left(\mathbf{q}_{\mathrm{m}}^{\mathrm{T}}, \mathbf{q}_{*}^{\mathrm{T}}\right)$ a precios mayorista $\mathbf{p}_{\mathrm{M}}^{\mathrm{T}}=\left(\mathbf{p}_{\mathrm{m}}^{\mathrm{T}}, \mathbf{p}_{*}^{\mathrm{T}}\right)$, con $\mathbf{q}_{\mathrm{k}}, \mathbf{p}_{\mathrm{k}} \in \mathbb{R}_{\geq 0}^{\mathbf{a}_{\mathrm{k}}}, \mathrm{k}=\{\mathrm{M}, \mathrm{m}, *\}$. Por otro lado, los factores productivos son la cantidad de leche cruda $\left(q_{1}^{d}\right)$ y otros factores $\left(\mathbf{z}_{\mathrm{M}}\right)$, con precios $\mathrm{p}_{1}$ y $\mathbf{w}_{\mathrm{M}}$, respectivamente. Para modelar la decisión de la firma representativa en un marco de multi-insumo y 2 productos, ${ }^{6}$ se asumen coeficientes de conversión exógenos (en el corto plazo) para cada conjunto de productos: $\Phi_{\mathrm{m}} \in \mathbb{R}_{\geq 0}^{\mathrm{a}_{\mathrm{m}}} \mathrm{y} \Phi_{*} \in \mathbb{R}_{\geq 0}^{\mathrm{a}_{\mathrm{s}}}$, entonces la oferta de la industria en litros equivalentes (Ite) es $\mathrm{q}_{\mathrm{m}}=\Phi_{\mathrm{m}}^{\mathrm{T}} \mathrm{q}_{\mathrm{m}} \mathrm{y}$ $\mathrm{q}_{*}=\Phi_{*}^{\mathrm{T}} \mathrm{q}_{*}$, respectivamente.

Con una tecnología de costos Cobb-Douglas para dos productos (Greer, 2012), $\mathrm{A}_{\mathrm{m}} \mathrm{q}_{\mathrm{m}}^{\mathrm{b}} \mathrm{q}^{\mathrm{c}}$, implica costos nulos si uno de los ellos no se produce, por lo que se propone una extensión de dicha tecnología de la siguiente manera,

$$
\begin{aligned}
\mathrm{C}\left(\mathrm{q}_{\mathrm{m}}, \mathrm{q}_{*}, \mathrm{p}_{\mathrm{l}}, \mathbf{w}_{\mathrm{m}}\right) & =\mathrm{A}_{\mathrm{m}} \mathrm{q}_{\mathrm{m}}^{\mathrm{b}} \mathrm{q}^{\mathrm{c}} \mathbf{1}_{\left\{\mathrm{q}_{\mathrm{m}}>0, \mathrm{q}_{*}>0\right\}}+\mathrm{B}_{\mathrm{m}} \mathrm{q}_{\mathrm{m}}^{\mathrm{b}^{\prime}} \mathbf{1}_{\left\{\mathrm{q}_{\mathrm{m}}>0, \mathrm{q}_{*}=0\right\}}+ \\
& +\mathrm{C}_{\mathrm{m}} \mathrm{q}^{\prime} \mathbf{1}_{\left\{\mathrm{q}_{\mathrm{m}}=0, \mathrm{q}_{>}>0\right\}},
\end{aligned}
$$

donde $b, c, b^{\prime}$ y $c^{\prime}$ son parámetros de la función de costos; $A_{m^{\prime}} B_{m}$ y $C_{m}$ funciones conocidas de éstos $\mathrm{y}$ de los precios de los insumos $\left(\mathrm{p}_{1}, \mathrm{w}_{\mathrm{M}}\right)$, siendo $\mathrm{w}_{\mathrm{M}}$ precios de insumos distintos a la leche, y $\mathbf{1}_{\{,\}}$la función indicadora. Asumiendo $\mathrm{q}_{\mathrm{m}}>0 \mathrm{yq} *>0$, el problema de la industria es la elección de la cantidad de litros que colocará en el mercado interno a los precios $\mathrm{p}_{\mathrm{M}}$, y en el externo a los precios $\mathrm{p}^{*}$, para maximizar $\pi\left(\mathrm{q}_{\mathrm{m}}, \mathrm{q}_{*}\right)=\mathrm{p}_{\mathrm{M}} \mathrm{q}_{\mathrm{M}}+\mathrm{p}_{*} \mathrm{q}_{*}--\mathrm{A}_{\mathrm{m}}\left(\mathrm{b}, \mathrm{c}, \mathrm{p}_{1}, \mathbf{w}_{\mathrm{m}}\right) \mathrm{q}_{\mathrm{m}}^{\mathrm{b}} \mathrm{q}^{\mathrm{c}}$. De la solución de este problema, las ofertas son

$$
\begin{aligned}
& \mathrm{q}_{\mathrm{m}}^{\mathrm{s}}=\lambda_{0, \mathrm{~m}}^{\mathrm{s}} \mathrm{A}_{\mathrm{m}}^{\frac{1}{1-b-c}} \mathrm{p}_{*}^{\frac{-c}{1-b-c}} \frac{\mathrm{c}-1}{\mathrm{p}_{\mathrm{M}}^{1-\mathrm{b}-\mathrm{c}}}, \\
& \mathrm{q}_{*}^{\mathrm{s}}=\lambda_{0, *}^{\mathrm{s}} \frac{1}{\frac{1}{1-b-c}} \mathrm{p}_{*}^{\frac{\mathrm{b}-1}{1-b-c}} \frac{-\mathrm{c}}{\mathrm{p}_{\mathrm{M}}^{1-\mathrm{b}-\mathrm{c}}}, \\
& \operatorname{con} \lambda_{0, \mathrm{~m}}^{\mathrm{s}}=\mathrm{c}^{\mathrm{c} /(1-\mathrm{b}-\mathrm{c})} \mathrm{b}^{(1-\mathrm{c}) /(1-\mathrm{b}-\mathrm{c})} \mathrm{y} \lambda_{0, *}^{\mathrm{s}}=\mathrm{c}^{(1-\mathrm{b}) /(1-\mathrm{b}-\mathrm{c})} \mathrm{b}^{\mathrm{b} /(1-\mathrm{b}-\mathrm{c})} .
\end{aligned}
$$

Por otro lado, para obtener la demanda de leche cruda, se especifica:

$$
A_{m}\left(b, c, p_{1}, w_{m}\right)=\Upsilon(b, c) p_{1}^{\delta_{1}} \prod_{k=1}^{n_{m}} w_{k}^{\delta_{k, w_{m}}} .
$$

Sustituyendo en la ecuación (9) y aplicando lema de Shephard en dicha función de costos respecto al precio de la leche cruda, se obtiene la demanda del insumo:

\footnotetext{
${ }^{6}$ Litros industrializados al mercado doméstico y al internacional.
} 


$$
\mathrm{q}_{l}^{\mathrm{d}}=\Upsilon(\mathrm{b}, \mathrm{c}) \prod_{\mathrm{k}=1}^{\mathrm{n}_{\mathrm{m}}} \mathrm{w}_{\mathrm{k}}^{\delta_{\mathrm{k}, \mathrm{w}_{\mathrm{m}}}} \delta_{\mathrm{l}} \mathrm{p}_{\mathrm{l}}^{\delta_{1}-1} \mathrm{q}_{\mathrm{m}}^{\mathrm{b}} \mathrm{q}_{*}^{\mathrm{c}}
$$

Usando la dependencia de los precios de las ecuaciones 9 y 10 , en $q_{1}^{d}$ se incluyen los argumentos $\mathrm{p}_{\mathrm{M}} \mathrm{y} \mathrm{p}^{*}$ de forma multiplicativa; mirando a la inversa, de la ecuación 13 a $\mathrm{q}_{\mathrm{m}}^{\mathrm{s}} \mathrm{y}$ $\mathrm{q}_{*}^{\mathrm{s}}$, las últimas dependerán de forma multiplicativa de los precios de los factores. Aplicando logaritmo en 13 y usando esta dependencia en precios, se obtiene la demanda log-lineal de la leche cruda, i.e., $\ln \mathrm{q}_{1}^{\mathrm{d}}=\lambda_{0,1}^{\mathrm{d}}+\prod_{\mathrm{k}=1}^{\mathrm{n}_{\mathrm{m}}} \delta_{\mathrm{k}, \mathrm{w}_{\mathrm{m}}} \ln \mathrm{w}_{\mathrm{km}}+\delta_{1}\left(\delta_{1}-1\right) \ln \mathrm{p}_{1}+\tau_{\mathrm{m}} \ln \mathrm{p}_{\mathrm{M}}+\tau_{*} \ln \mathrm{p}_{*}$.

En el nivel de comercialización interna, los bienes son distribuidos en el mercado por agentes que demandan al nivel mayorista los productos como insumos. Aunque no sufren una transformación física, se produce un servicio de distribución, i.e. una función de producción ( $\mathrm{f}_{\mathrm{m}}$ ). Suponiendo una tecnología Cobb-Douglas, la función de producción es $\mathrm{f}_{\mathrm{m}}\left(\mathrm{q}_{\mathrm{m}}, \mathbf{z}_{\mathrm{f}}\right)=\mathrm{D}_{\mathrm{m}} \mathrm{q}_{\mathrm{m}}^{\beta_{\mathrm{m}}} \prod_{\mathrm{j}=1}^{\mathrm{n}_{\mathrm{f}}} \mathrm{z}_{\mathrm{jf}}^{\beta_{\mathrm{f}}}$, donde $\mathrm{q}_{\mathrm{m}}$ son productos lácteos (Ite) que compra a los mayoristas, $\mathrm{z}_{\mathrm{ij}}$ son los restantes $n_{\mathrm{f}}$ factores productivos y $\mathrm{D}_{\mathrm{m}}$ es una constante tecnológica. Si vende al mercado interno a los precios $\mathrm{p}_{\mathrm{M}}$, pagando $\mathrm{p}_{\mathrm{M}}$ en el mercado mayorista $\mathrm{y} \mathrm{w}_{1 \mathrm{f}}, \ldots, \mathrm{w}_{\mathrm{n}_{\mathrm{f}}} \mathrm{f}$ por los otros factores, de la maximización de los beneficios del distribuidor se obtiene la demanda de productos lácteos:

$q_{m}^{d}=G_{m}\left(D_{m}, \beta_{m}, \beta_{1 f}, \ldots, \beta_{n_{f}}\right) p_{M} e^{\left(-1+\sum_{j} \beta_{j f}\right) / \psi} p_{m} e^{1 / \psi} \prod_{j} w_{j f} e^{\beta_{j f} / \psi}$,

con $G_{m}\left(D_{m}, \beta_{m}, \beta_{1 f}, \ldots, \beta_{n_{f} f}\right) \equiv D_{m} e^{1 / \psi} \beta_{m} e^{1-\sum_{j} \beta_{j f} / \psi} \prod \beta_{j f} e^{\beta_{j f} / \psi}$ y $\psi=1-\beta_{m}-\sum_{j} \beta_{j f}$. A su vez, la oferta de estos distribuidores minoristas a los consumidores finales queda explicitada como

$\mathrm{q}_{\mathrm{cf}}^{\mathrm{s}}=\mathrm{K}_{\mathrm{m}}\left(\mathrm{D}_{\mathrm{m}}, \beta_{\mathrm{m}}, \beta_{1 \mathrm{f}}, \ldots, \beta_{\mathrm{n}_{\mathrm{f}} \mathrm{f}}\right) \mathrm{p}_{\mathrm{M}} \mathrm{e}^{\beta_{\mathrm{m}} / \psi} \mathrm{p}_{\mathrm{m}} \mathrm{e}^{\beta_{\mathrm{m}}+\sum_{\mathrm{j}} \beta_{\mathrm{jf}} / \psi} \prod_{\mathrm{j}} \mathrm{w}_{\mathrm{if}} \mathrm{e}^{\beta_{\mathrm{if}} / \psi}$,

donde $\mathrm{K}_{\mathrm{m}}\left(\mathrm{D}_{\mathrm{m}}, \beta_{\mathrm{m}}, \beta_{1 \mathrm{f}}, \ldots, \beta_{\mathrm{n}_{\mathrm{f}} \mathrm{f}}\right) \equiv \mathrm{D}_{\mathrm{m}} \mathrm{e}^{1 / \psi} \beta_{\mathrm{m}} \mathrm{e}^{\beta_{\mathrm{m}} / \psi} \prod_{\mathrm{j}} \beta_{\mathrm{jf}} \mathrm{e}^{\beta_{\mathrm{if}} / \psi}$.

La demanda final de los consumidores se supone una demanda tradicional log-log (Barnett y Serletis, 2008),

$\ln \mathrm{q}_{\mathrm{cf}}^{\mathrm{d}}=\eta_{0, \mathrm{cf}}+\eta_{\mathrm{I}} \ln \mathrm{I}+\eta_{\mathrm{m}, \mathrm{cf}} \ln \mathrm{p}_{\mathrm{m}}+\sum_{\mathrm{j}} \eta_{\mathrm{j}, \mathrm{cf}} \ln \mathrm{p}_{\mathrm{j}}$

con / como ingreso, $\eta_{\mathrm{I}}=\partial \ln \mathrm{q}_{\mathrm{cf}}^{\mathrm{d}} / \partial \ln \mathrm{I}$ es la elasticidad ingreso $\mathrm{y}, \eta_{\mathrm{m}, \mathrm{cf}}$ la precio-propio no compensada (Cournot). Por lado de la demanda externa ( $\mathbf{q}_{*}^{\mathrm{d}}$ ) se asume que está dada, y queda representada por el precio internacional, a saber, $\mathbf{p}_{*}$.

\section{Anexo 2. Derivación del Modelo Econométrico}

Se utiliza las versiones linealizadas de las ecuaciones de oferta-demanda detalladas en el Anexo 1, incorporando la dimensión temporal de las variables. Comenzando desde el nivel primario de la cadena, en Argentina suelen producirse eventos climáticos extremos e intervención política. Ambos ocurrieron en el período, y la intervención se produjo a partir de la aplicación de compensaciones al productor. Así, estos factores exógenos se incorporan en la oferta de leche cruda (ecuación 8) por medio de $\mathrm{B}_{\mathrm{lt}}$. Sea Comp $_{\mathrm{t}}$ una variable binaria si el productor recibe compensaciones; $\mathrm{Clima}_{\mathrm{t}}$ también binaria sobre las condiciones climáticas extremas (inundaciones o sequías); $\mathrm{E}_{(.)}$son variables estacionales trimestrales y el efecto aleatorio $\mathrm{e}_{\mathrm{lt}}^{\mathrm{s}}$ :

$\mathrm{B}_{\mathrm{lt}}=\exp \left(\alpha_{0,1}+\alpha_{1,1} \operatorname{Comp}_{\mathrm{t}}+\alpha_{2,1} \mathrm{Clima}_{\mathrm{t}}+\sum_{\mathrm{k}=2}^{4} \vartheta_{\mathrm{k}, 1} \mathrm{E}_{\mathrm{k}}+\mathrm{e}_{\mathrm{lt}}^{\mathrm{s}}\right)$. 
Por otra parte, la cuenca lechera de Argentina se encuentra en una zona que compite con otras producciones, principalmente la soja. Así, se utiliza el precio de ésta ( $\mathrm{p}_{\mathrm{t}}^{\text {soja }}$ ) como costo de oportunidad. Y se completa la oferta de leche con el precio del maíz ( $\mathrm{p}_{\mathrm{t}}^{\text {maiz }}$ ) como costo productivo en la alimentación del ganado.

En el nivel industrial, se incluyen costos de producción: a. de mano de obra ( salario $^{\text {Ind }}$ ), b. de procesar la leche cruda (de la ecuación 12 del Anexo 1, la tecnología queda definida por q lt ) y, c. otros insumos, aproximados por el Índice de Precios Internos Mayorista ( $\operatorname{IPIM}_{t}^{\mathrm{NG}}$ ). Además, se incluyen los niveles de inversión en la industria (Inversión Bruta Interna Fija, IBIF $)$. Todas se incorporan en la ecuación 10 del Anexo 1: $\lambda_{0, \mathrm{~m}} \mathrm{~A}_{\mathrm{m}}^{\frac{1}{1-\mathrm{b}-\mathrm{c}}}=\exp \left(\alpha_{0, \mathrm{~m}}++\alpha_{1, \mathrm{~m}} \ln \operatorname{IBIF}_{\mathrm{t}}+\alpha_{2, \mathrm{~m}} \mathrm{IPIM}_{\mathrm{t}}^{\mathrm{NG}}+\alpha_{3, \mathrm{~m}} \ln\right.$ salario $\left._{\mathrm{t}}^{\text {Ind }}+\alpha_{4, \mathrm{~m}} \ln \mathrm{q}_{\mathrm{lt}}+\mathrm{e}_{\mathrm{mt}}^{\mathrm{s}}\right)$, con $\mathrm{e}_{\mathrm{mt}}^{\mathrm{s}}$ como componente aleatorio.

Se toma el precio internacional p* dado. Además, el sector exportador argentino tiene dos características: a. suele haber intervención por parte del estado medio de retenciones (se incluye una variable dummy "Ret", para discriminar los períodos donde esta política esta vigente), b. se exportan cantidades muy inferiores a las que se destinan al mercado interno por lo que las exportaciones dependen de las cantidades disponibles de materia prima. Incorporando en la ecuación 12, a saber, $\lambda_{0, *} \mathrm{~A}_{\mathrm{m}}^{\overline{1-b-c}}=\exp \left(\alpha_{0, *}++\sum_{\mathrm{k}=2}^{4} \vartheta_{\mathrm{k}, *} \mathrm{E}_{\mathrm{k}}+\alpha_{1, *} \operatorname{Ret}_{\mathrm{t}}+\alpha_{2, *} \ln \mathrm{q}_{\mathrm{lt}}+\mathrm{e}_{*_{\mathrm{t}}}^{\mathrm{s}}\right)$, con $\mathrm{e}_{*_{\mathrm{t}}}^{\mathrm{s}}$ aleatorio.

La demanda derivada de leche del nivel industrial se parametriza con los precios explícitos (ecuación 13 del Anexo 1) y $\gamma_{0,1}^{\mathrm{d}}+\sum_{\mathrm{k}=1}^{\mathrm{n}_{\mathrm{m}}} \delta_{\mathrm{k}, \mathrm{w}_{\mathrm{m}}} \ln \mathrm{w}_{\mathrm{km}}=\alpha_{0,1}^{\mathrm{d}}+\alpha_{1,1}^{\mathrm{d}} \mathrm{t}+\mathrm{e}_{\mathrm{lt}}^{\mathrm{d}}$ y $\tilde{\delta}_{1}=\delta_{1}\left(\delta_{1}-1\right)$. Sólo se utiliza una tendencia para identificar la función, por falta de mayor información de variables de demanda intermedia.

En la demanda minorista (ecuación 14 del anexo 1) se agrega el Estimador Mensual de Actividad Económica ( $\mathrm{EMAE}_{\mathrm{t}}$ ) para tener en cuenta la demanda interna. La versión lineal de 14 se parametriza: $\lambda_{0, \mathrm{~m}}^{\mathrm{d}}+\sum_{\mathrm{j}} \theta_{\mathrm{jf}}^{\mathrm{d}} \ln \mathrm{w}_{\mathrm{jf}}=\alpha_{0, \mathrm{~m}}^{\mathrm{d}} \mathrm{EMAE}_{\mathrm{t}}+\mathrm{e}_{\mathrm{mt}}^{\mathrm{d}}$, con $\mathrm{e}_{\mathrm{mt}}^{\mathrm{d}}$ aleatorio.

En la oferta al consumidor final (además $\mathrm{p}_{\mathrm{mt}}$ ) se considera a. $\mathrm{p}_{\mathrm{Mt}}$ como costo de adquisición; $b$. el Índice de Precios al Consumidor ( $\mathrm{e}_{\mathrm{cf}_{\mathrm{t}}}^{\mathrm{s}}$ ) para otros costos del proceso de distribución y $\mathrm{e}_{\mathrm{cf}_{\mathrm{t}}}^{\mathrm{s}}$ aleatorio. Así, la parametrización de la ecuación 15 linealizada es $\lambda_{0, \mathrm{cf}}^{\mathrm{s}}+\sum^{\mathrm{j}} \theta_{\mathrm{jf}}^{\mathrm{s}} \ln \mathrm{w}_{\mathrm{jf}}=\alpha_{0, \mathrm{cf}}^{\mathrm{s}}+\alpha_{1, \mathrm{cf}}^{\mathrm{s}} \mathrm{IPC}_{\mathrm{t}}^{\mathrm{NG}}+\mathrm{e}_{\mathrm{cf}_{\mathrm{t}}}^{\mathrm{s}}$. En el caso de la demanda final (ecuación 16 del Anexo 1) se consideran las variables explícitas, una la estacionalidad de consumo $\left(\mathrm{E}_{\mathrm{cf}}\right) \mathrm{y}$, el efecto aleatorio $\mathrm{e}_{\mathrm{cf}_{\mathrm{t}}}^{\mathrm{d}}$, por medio de $\eta_{0, \mathrm{cf}}=\alpha_{0, \mathrm{cf}}+\alpha_{1, \mathrm{cf}} \mathrm{E}_{\mathrm{cf}_{\mathrm{t}}}+\mathrm{e}_{\mathrm{cf}_{\mathrm{t}}}^{\mathrm{d}}$.

\section{Anexo 3. Manipulación de las Variables}

Conversión a Litros Equivalentes: Como en el proceso de industrialización se obtienen una multiplicidad de productos, en el presente trabajo se realiza una homogeneización a partir de coeficientes de conversión para transformarlos a litros equivalentes (Ite). Las fuentes de éstos son:7 MiniAgri, Oficina Nacional de Control Agropecuario (ONCCA) y Instituto Nacional de Tecnología Industrial (INTI). Se opera un promedio de éstas, y el valor final seleccionado sobre la base de consultas a expertos de la industria láctea. Dichos coeficientes, medidos en litros (Its) por kilogramo (kg), son: 4.04 para crema, 13.4 para queso pasta dura, 10 para queso pasta semidura, 8.06 para queso pasta blanda y fresco; 10.3 para manteca, 8.32 para leche en polvo entera y 12.5 para descremada, 4.2 en dulce de leche y 0.92 para yogur.

Precios mayoristas, de exportación y minorista: Sea $\mathrm{q}_{\mathrm{jt}}$ la cantidad total (Ite) colocadas en el mercado $j$, con $\mathrm{j}=\{\mathrm{m}, *\}$, en el momento $t$, $\mathrm{q}_{\mathrm{ijt}}$ la cantidad correspondiente al $i$-ésimo producto en Ite; entonces, las ponderaciones del $j$-ésimo mercado es $\omega_{\mathrm{ijt}}=\mathrm{q}_{\mathrm{ijt}} / \mathrm{q}_{\mathrm{jt}}$, y el índice de precios del eslabón $\mathrm{w}\left(\mathrm{w}=\mathrm{M}, \mathrm{m},{ }^{*}\right)$ es $\mathrm{p}_{\mathrm{wt}}=\sum_{\mathrm{i}=1}^{\mathrm{a}_{\mathrm{j}}} \omega_{\mathrm{ijt}} \mathrm{p}_{\mathrm{iwt}}$, con $\mathrm{a}_{\mathrm{j}}$ la cantidad total de bienes en el mercado $j$, $\mathrm{p}_{\mathrm{iwt}}$ el precio del bien $i$ en $\$ /$ te, y $\omega_{\mathrm{ijt}}$ es idéntico en el eslabón minorista y mayorista por los supuestos de partida, en ambos casos los productos lácteos coinciden (no sus precios).

7 Aunque algunas de ellas ya no existen, la información está disponible en la web. 
Estacionalidades: Las variables estacionales se construyen a partir de un análisis de la estacionalidad observada en la producción y consumo. Así, en la producción primaria y las cantidades exportadas tienen valores superiores a su media general entre agosto-enero, por lo que se crean cuatro variables indicadoras: $E_{1}$ para los meses de nov a ene, $E_{2}$ de feb a abr, $\mathrm{E}_{3}$ de may a jun $\mathrm{y}, \mathrm{E}_{4}$ de ago a oct.

A nivel mayorista no se observa un patrón estaciona y, en el nivel de consumo final, estacionalidad alta en marzo ${ }^{8}$ y diciembre. ${ }^{9}$ Por ello, se modela mediante una variable indicadora, $\mathrm{E}_{\mathrm{cf}}$, igual a uno en marzo y diciembre, y cero en el resto del año.

8 Consultado con expertos y agentes en el área, quienes indicaron que en marzo comienza el ciclo lectivo y esto afecta la demanda de lácteos.

9 En épocas de las fiestas de fin de año. 